\title{
CHANGE IN FIELD HARMONICS AFTER QUENCH AND THERMAL CYCLES IN SUPERCONDUCTING MAGNETS*
}

\author{
R. Gupta, A. Jain, J. Muratore, P. Wanderer, E.Willen, BNL, Upton, NY 11973 USA and \\ C. Wyss, CERN, Geneva, Switzerland
}

\section{Abstract}

A change in field harmonics after quench and thermal cycles has been observed in superconducting magnets for the Relativistic Heavy Ion Collider (RHIC). This paper presents the results of a systematic investigation of this effect in a number of RHIC dipole and quadrupole magnets. These changes in field harmonics may limit the ultimate field quality and its reproducibility in superconducting magnets. A change in pre-stress has also been observed after quench and thermal cycles. A possible link between these two changes is explored.

\section{INTRODUCTION}

To satisfy high field quality requirements in RHIC interaction region (IR) quadrupoles, a magnetic tuning shim method is used to compensate harmonic errors after measurements [1]. However, it has been observed that the harmonics changes after quenches and thermal cycles are larger than the capability of the tuning shim correction. The stability and resolution of the measurement system is known to be sufficient for the observations. In the following sections we present the results of a systematic study made to understand and quantify this effect.

The skew $\left(a_{n}\right)$ and normal $\left(b_{n}\right)$ components of field harmonics are defined as follows :

$$
B_{y}+i B_{x}=10^{-4} B_{R} \sum_{n=0}^{\infty}\left[b_{n}+i a_{n}\right]\left[\frac{x+i y}{R}\right]^{n},
$$

where $B_{x}$ and $B_{y}$ are the components of the field at $(x, y)$ and $B_{R}$ is the magnitude of the field of the fundamental harmonic at a reference radius $R$.

\section{CURRENT DEPENDENCE}

During the calibration runs to obtain the harmonics produced by tuning shims at the design field, it was discovered that two runs produced two different harmonics. The magnetization of the tuning shim iron was ruled out as a possible source because the difference in harmonics between two runs was nearly the same at all currents. To confirm this inference, the tuning shims were removed and the measurements were repeated with a number of interleaving quenches and thermal cycles. Fig. 1 shows the variation in integral $b_{5}$ at $40 \mathrm{~mm}$ reference radius as a function of current during six up ramps in $130 \mathrm{~mm}$ aper- ture RHIC IR quadrupole QRK102. Each ramp was preceded by either a quench or a thermal cycle or both. The $b_{5}$ change is independent of current. This eliminates yoke saturation and persistent currents as possible sources as both would produce a current dependent change. A similar current independent change is seen in other harmonics as well. This observation indicates that the change in harmonics is related to the change in magnet geometry.

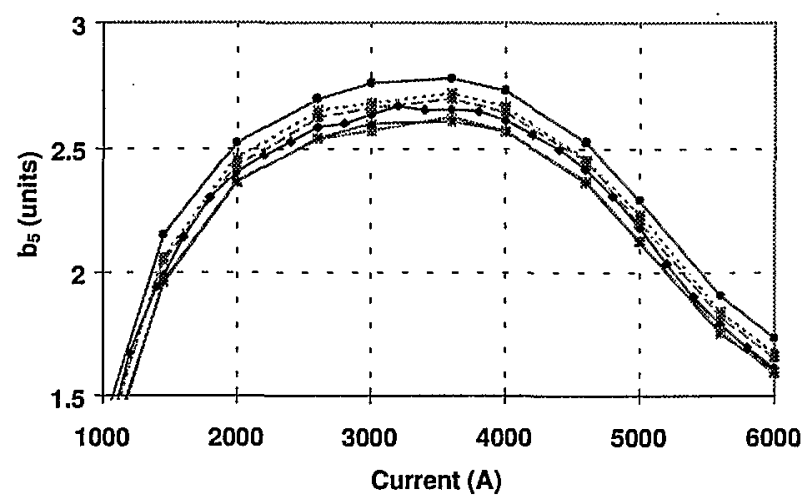

Fig. 1 : The current dependence in $b_{5}$ during six up ramps (separated by quench and/or thermal cycles) in 3.4 $\mathrm{m}$ long $130 \mathrm{~mm}$ aperture RHIC IR quadrupole QRK102.

\section{HARMONIC CHANGES IN DIPOLES}

The mechanical design and assembly of RHIC quadrupoles are similar to those of the dipoles. For example, the quadrupole yoke and collaring scheme has a two fold symmetry. Therefore, the dipoles may also show a corresponding change in harmonics. This indeed was found in both $80 \mathrm{~mm}$ aperture and $100 \mathrm{~mm}$ aperture dipoles in terms of the observed changes in $a_{1}$, the skew quadrupole harmonic, which is related to up-down asymmetry.

Fig. 2 shows a significant change in integral $a_{1}$ at 31 $\mathrm{mm}$ reference radius in RHIC $100 \mathrm{~mm}$ aperture insertion dipole DRZ106 after a number of quenches and thermal cycles. No change was, however, observed in repeated up and down ramps (dc loops) which is important for a stable accelerator operation. "LN2 Run" are the measurements in the range of 20-90 K and represent the change in geometry due to cooldown. Persistent current induced harmonics are not expected as the cable is in a non-superconducting state. Warm harmonics return to their original values. This has been seen in many (but not all) magnets.

\footnotetext{
${ }^{*}$ Work supported by U.S. Department of Energy under contract No. DE-ACO2-98CH10886.
} 


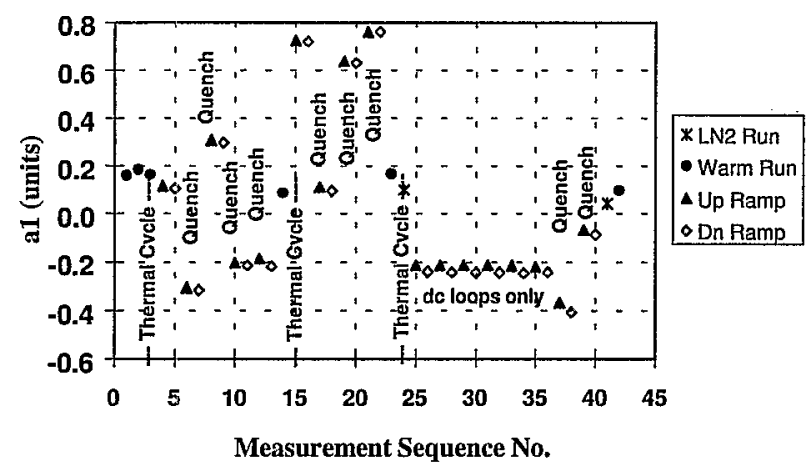

Fig. 2 : Variation in integral $a_{1}$ at $31 \mathrm{~mm}$ reference radius in RHIC $100 \mathrm{~mm}$ aperture dipole DRZ106 from quench and thermal cycles.

\section{AXIAL DEPENDENCE OF CHANGE}

In Fig. 3, we show the measured $a_{1}$ before and after a thermal cycle (thin lines) and the change between the two (thick line) in five sections of magnet DRZ106. This axial scan was done to determine if the changes are lumped locally or distributed uniformly. The large $a_{1}$ in the first position is from the lead end. The change in harmonic is about the same at all locations. This means that the coil as a whole is changing its geometry.

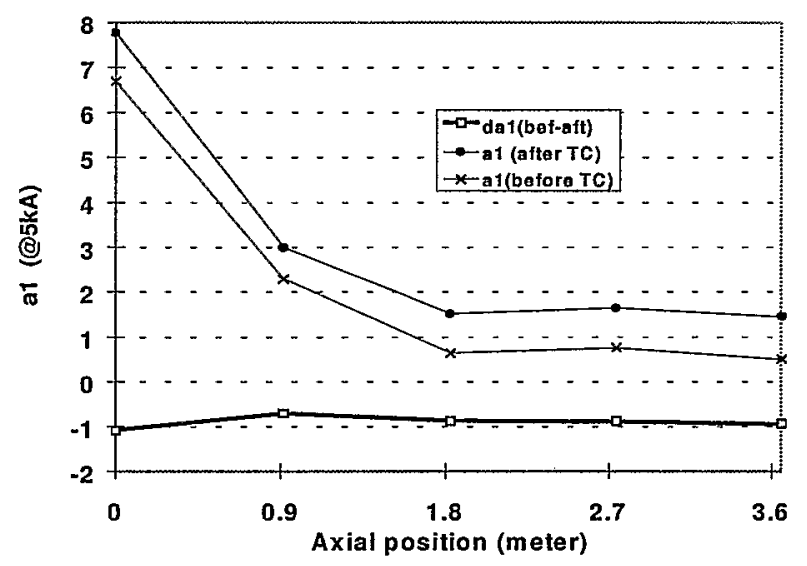

Fig. 3 : Axial dependence of the change in $a_{1}(@ 31 \mathrm{~mm}$ ) caused by a thermal cycle in DRZ106.

\section{CHANGE IN A SERIES OF MAGNETS}

In this section the results of measurements in eight 1.44 meter long $130 \mathrm{~mm}$ aperture Q1 magnets are presented to statistically quantify this effect. Since the issue is most critical in IR quadrupoles a number of these magnets were measured before and after quench and thermal cycles. Fig. 4 shows the change in integrated harmonics from quench and Fig. 5 from thermal cycle. The solid line represents the mean and the dashed line one sigma around the mean. One can see that generally a thermal cycle generates smaller changes than a quench. The magnitudes of the observed changes in harmonics are acceptable in RHIC [2].
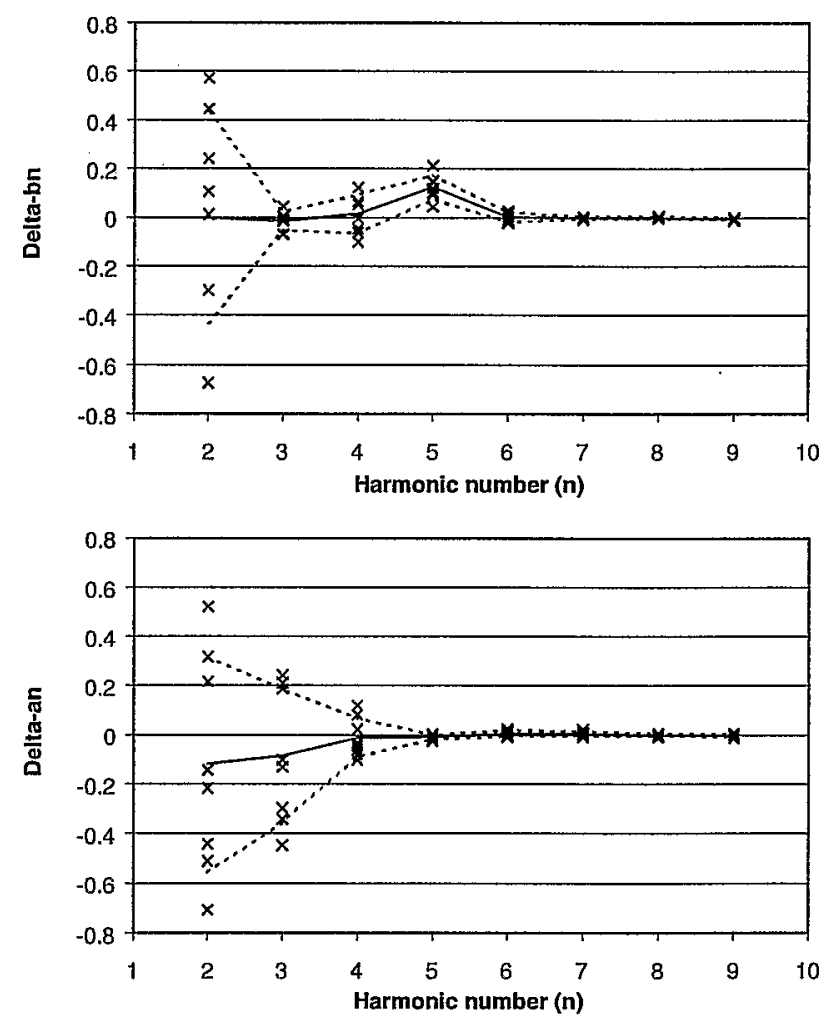

Fig. 4 : Change in harmonics in eight $130 \mathrm{~mm}$ aperture insertion quadrupoles caused by a quench.
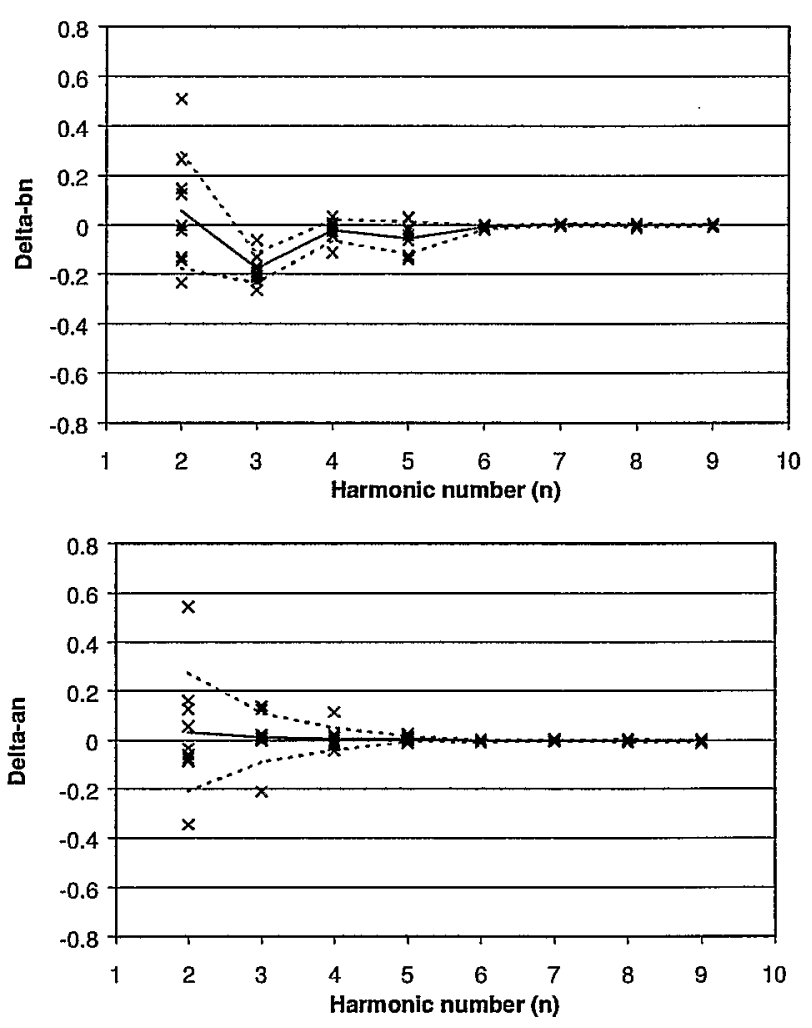

Fig. 5 : Change in harmonics in eight $130 \mathrm{~mm}$ aperture insertion quadrupoles caused by a thermal cycle. 
For quenches (Fig. 4), the changes are of random nature except for a small systematic $b_{5}$ (the first allowed harmonic in quadrupoles). A similar behavior is also seen in dipoles where the change in all harmonics except for a small systematic $b_{2}$ (the first allowed harmonic in dipoles) is of a random nature.

In the case of thermal cycles (Fig. 5), the changes are of a random nature except for a systematic $b_{3}$ (always a negative change). The magnet QRI105 was subjected to five thermal cycles to further study $b_{3}$. The net drop stabilized at 0.3 units after three thermal cycles meaning that the collared coil geometry stabilized. This magnet had showed small change in other harmonics ( $\sim 0.1$ unit or less) after first thermal cycle. The change remained small $(\sim 0.1$ unit or less) during the remaining thermal cycles.

\section{CHANGES IN AZIMUTHAL STRESS}

A change in azimuthal coil stress on pole faces has also been observed after quench and thermal cycles. We examine the change in azimuthal stress as independent evidence of the change in coil geometry. In Fig. 6, we have plotted the azimuthal coil stress on four pole faces (dashed lines) after successive quenches together with a thermal cycle in between in $100 \mathrm{~mm}$ aperture dipole DRZ105. The magnet was cold but not powered. We have also plotted the average stress on four pole faces (solid line) and the difference between the average of top two and bottom two (thick solid line). Though the absolute values of these stresses (measured by strain gauges) may not be reliable to a few hundred psi, the relative change is. One can clearly see a change in behavior after each quench. Most interesting is the difference between top and bottom (thick solid line) as this is related to change in $a_{1}$ harmonic (Fig. 2 and Fig. 3). A one to one correlation can not be established between $a_{1}$ and stress as the stress measurements were done in DRZ105 and the magnetic measurements in DRZ106. However, both the harmonic and stress changes can be explained, depending on the computer models, by a geometric change which is of the order of 0.001 "' $\mu \mathrm{m})$.

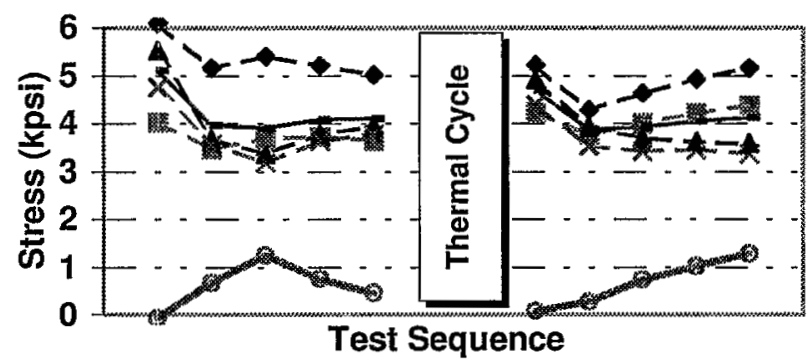

Fig. 6 : The azimuthal stress on pole faces (dashed lines), average (solid) and difference between top and bottom (thick solid line) after successive quenches together with a thermal cycle in between in the magnet DRZ105.

\section{DISCUSSION}

In addition to the $130 \mathrm{~mm}$ aperture quadrupole and 100 $\mathrm{mm}$ aperture dipole magnets, harmonic changes of similar magnitude have also been observed in $80 \mathrm{~mm}$ aperture dipole and $80 \mathrm{~mm}$ aperture quadrupole magnets for RHIC. All these magnets were made using a similar design and construction techniques. Therefore, it implies that it is a common phenomenon in such magnets. These changes are larger than the measurement uncertainties. The changes are smaller than the RMS variations in harmonics in a series of magnets. The effect, though of smaller magnitude, has also been observed in SSC dipole magnets [3]. The SSC design was based on stainless steel collars, whereas the RHIC magnets use the yoke as a collar and phenolic spacers (which are not as strong as stainless steel) between coil and yoke. Though this is an important difference, we can not say definitely that this is the sole reason for smaller changes in SSC dipole harmonics. SSC magnets also had a smaller aperture. Moreover, we have examined this effect in only one magnet.

The following mechanism may explain the observed changes. Quenches and thermal cycles generate a pressure shock which may cause a change in the internal geometry of the collared coil by a small amount $(\sim 25 \mu \mathrm{m})$. Unless overcome by large mechanical forces (such as another shock), this small change may get locked into a new and slightly different coil geometry, particularly when the magnet is cold and the stresses are small. A uniform change in the $a_{1}$ harmonic along the magnet length (see Fig. 3) means that effectively the coil midplane moves as a whole.

In most cases (but not all), the harmonics returned to their original values when the magnet was warmed up to room temperature. The azimuthal stresses are much larger when the magnet is warm as compared to when it is cold. It is possible that larger azimuthal stresses at room temperature are enough to overcome the friction to bring the magnet back to its original geometry.

\section{CONCLUSIONS}

The ultimate field quality in superconducting magnets may be limited by the dynamic changes caused by quench and thermal cycles. These changes are much larger than the resolution of tuning shims and as such can not be compensated by the static nature of tuning shim correction. The measured harmonic changes, though significant; are acceptable in RHIC magnets.

\section{REFERENCES}

[1] R. Gupta, et al., "Tuning Shims for High Field Quality in Superconducting Magnets", Proc. of MT-14, IEEE Transaction on Magnetics, Vol. 32, No. 4, July 1996.

[2] S. Peggs and J. Wei, private communication.

[3] P. Wanderer, et al., "Partial Lifetime Test of an SSC Collider Dipole", MT-13, Sep. 1993. 